\section{Les business models dans la distribution :}

\section{repérer les chemins de la performance}

\section{Pierre Volle}

Professeur, Université Paris - Dauphine

DRM - DMSP, UMR CNRS nº 7088

\section{Delphine Dion}

Maître de Conférences, IAE de Paris

GREGOR

Marie-Louise Heliès-Hassid

Maître de Conférences, Université Paris - Dauphine

DRM - DMSP, UMR CNRS n 7088

Avec la participation de Stéphane Sabbah

Directeur financier, SFD Espace SFR

\section{Les business models dans la distribution :}

\section{repérer les chemins de la performance}

\section{Résumé}

Malgré un usage répandu dans le secteur du commerce, le concept de business model (BM) reste largement à préciser. L'objectif de cet article est donc de mettre en évidence les éléments constitutifs d'un BM dans la distribution puis d'en dresser une première typologie. Nous sommes amenés à distinguer un BM qui consiste à simplifier la proposition de valeur tout en diminuant les coûts pour gagner en efficience, et un BM qui consiste à enrichir la proposition de valeur pour gagner en attractivité « par le haut ».

Mots-clés : distribution, commerce, stratégie, modèle d’affaires, business model

\section{Abstract}

In spite of a widespread usage among retail professionals, the concept of business model (BM) deserves some clarifications. Building on this point, the objective of our research is to shed some light on the constituents of BM's in the retail trades, and to offer a first typology.

Key words: retail, strategy, business model 


\section{Introduction}

Le concept de business model (modèle d'affaires) est utilisé par les professionnels de la distribution depuis la fin des années 90, essentiellement dans le secteur du commerce électronique. Il est désormais largement employé par l'ensemble des professionnels, y compris des acteurs bricks and mortar (les distributeurs traditionnels qui gèrent des chaines de magasins).

Le business model doit permettre de répondre à la question : « comment vais-je gagner de l’argent » ? Définir un business model revient donc à déterminer les sources de revenus que l'entreprise va tenter de générer (revenue model), les ressources et compétences qu'elle va mobiliser pour ce faire, les produits et services qu'elle va offrir, les activités qu'elle va ou non réaliser dans la chaîne de valeur et plus largement, la position qu'elle souhaite occuper dans le réseau de valeur du secteur (producteur, assembleur, commerçant, simple intermédiaire...).

Le concept de BM est très utile dans le sens ou de nombreuses questions de stratégie marketing en dépendent, notamment : qui sont les clients ? Comment peut-on se rendre utile à leurs yeux (proposition de valeur) ? Comment le marché est-il couvert, en termes symboliques (publicité) et physiques (distribution) ? Comment les clients sont-ils conquis et fidélisés ? Le concept de business model vient compléter les notions classiques de la stratégie d'entreprise, tels que les facteurs clés de succès ou l'avantage concurrentiel. Il permet d'envisager de nouvelles sources de revenus, d'identifier de nouvelles parties prenantes et d'élargir la vision que l'entreprise a de son client (Lecocq et al., 2006).

Cet article se donne pour objectif d'identifier les différents business models dans le secteur du commerce, puis de s'interroger sur leur efficience. Plus précisément, il s'agira d'identifier les éléments que les distributeurs peuvent combiner pour construire leurs business models (partie 1), puis de proposer une typologie de business models dans la distribution et d'en préciser l'agenda stratégique (partie 2).

Précisons que dans cet article, nous nous intéressons exclusivement aux distributeurs bricks and mortar - qui peuvent par ailleurs avoir développé une activité complémentaire sur catalogue ou en ligne - mais que les business models électroniques sont en dehors de notre périmètre ${ }^{2}$. Précisons aussi que l'on s'intéresse aux commerçants - ceux qui réalisent des actes de commerce de type achat / revente - et non pas à l'ensemble de intermédiaires du commerce (agents, courtiers...). Pour finir, seules les activités commerciales à destination des clients particuliers sont envisagées (B-to-C), la distribution professionnelle n'étant donc pas évoquée directement.

\section{1 - Les éléments constitutifs d'un BM dans la distribution}

L'objectif de cette première partie est de mieux caractériser les éléments d'un business model (BM) dans la distribution : ressources et compétences, offres et activités, sources de revenus et de charges. De nombreux observateurs seraient tentés de conclure que l'activité de revente de marchandises, avec la capacité à bien acheter comme compétence fondamentale, constitue l'essentiel d'un BM dans la distribution. De fait, si cette activité centrale constitue bel et bien la partie historique du commerce, les BM dans la distribution sont aujourd'hui nettement plus variés. La marge réalisée sur la revente de marchandises constitue une source de revenus parmi d'autres; la compétence à bien acheter doit être complétée par de nombreuses autres compétences. Dans cette partie, notre objectif consiste donc à montrer la diversité et la richesse des éléments constitutifs des BM dans le secteur du commerce.

\section{Les ressources et compétences exploitées par les distributeurs}

Les ressources - Les distributeurs s'appuient sur un ensemble de ressources variées: logistiques, commerciales, productives. Pendant longtemps, ils ont avant tout développé les ressources logistiques (entrepôts et plateformes, flotte de camions) et les espaces en front de vente (vitrines, surfaces de vente, gondoles, parkings, galeries marchandes...). Certains distributeurs sont issus de la fonction de grossiste, avec une forte dimension logistique (comme Promodès, racheté en 1999 par Carrefour). D’autres ont simultanément développé les ressources logistiques et le front de vente. Par exemple, Les Mousquetaires - qui regroupe les enseignes Intermarché, Ecomarché, Netto, Restaumarché, Véti, Roady et Bricomarché possède 46 bases logistiques (soit 1,3 million de $\mathrm{m}^{2}$ de stockage) et 2300 semi-remorques. Parallèlement, le groupe gère environ 4000 points de vente en Europe, pour une surface de vente dépassant 2 millions de mètres carrés.

${ }^{2}$ Pour une analyse des business models dans le commerce électronique, se référer à l’ouvrage de Isaac et Volle, (2008). 
Les distributeurs s'appuient également sur des capacités de production parfois importantes. Ainsi, le groupe Les Mousquetaires a poursuivi sa stratégie d'intégration en amont, à travers des participations dans plus d'une quarantaine de PME de production (flotte de bateaux de pèche, conserverie, embouteillage...).

Au-delà des ressources physiques, les BM dans la distribution s'appuient sur un ensemble de ressources intangibles désormais essentielles : marques (marque-enseignes et marques de distributeurs) à la fois vecteurs de trafic, de confiance et de marge ; systèmes d'information performants qui permettent à la fois de collecter des données clients et d'organiser les flux d'échanges avec les fournisseurs.

Les compétences - Dans le passé, le partage des rôles entre producteurs, intermédiaires et détaillants était simple. Aujourd'hui, afin d'optimiser les flux de marchandises et d'information, les producteurs et les distributeurs ont été amenés à redéfinir leur champs d'action: quasiment chaque fonction de distribution peut être assurée par chacun des membres du canal, ce qui impose aux distributeurs de développer fortement leurs compétences.

Figure 1 - L'évolution des canaux (d'après Mc Goldrick, 2003)

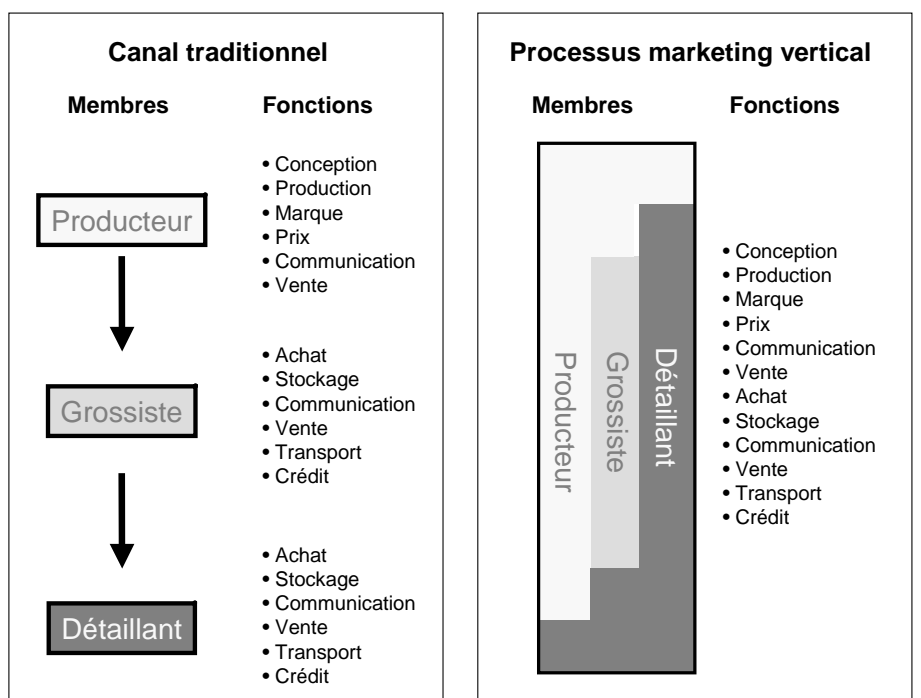

Pour faire face à ces nouveaux enjeux et pour exploiter pleinement les ressources dont ils disposent, les distributeurs ont développé un ensemble de compétences aujourd'hui complexes à acquérir ou à copier. L'activité de revente étant consubstantielle au commerce, les compétences centrales portent certainement sur les achats, notamment le référencement des fournisseurs et la négociation, qui permet d'acheter aux meilleures conditions et d'allonger les délais de paiement... Les exigences de traçabilité et de qualité, couplées à l'augmentation des achats à l'étranger, induisent un renforcement des compétences, notamment la spécification des cahiers des charges et le contrôle de la qualité.

Le développement des partenariats avec les industriels nécessite également un renforcement des compétences relationnelles avec la coordination des lancements ou le management des catégories de produits. Les distributeurs développent également des produits à leur marque, ce qui suppose de concevoir, de coordonner des sous-traitants, voire de produire. Un autre bloc de compétences porte sur la gestion des flux physiques et des flux d'information. Compétences aujourd'hui essentielles dans la mesure où la baisse des stocks et la limitation simultanée des ruptures constituent deux contraintes fortes mais contradictoires.

Les compétences liées aux espaces commerciaux sont également fondamentales ; elles portent à la fois sur la dimension immobilière (acheter des terrains, construire des bâtiments, louer et valoriser les surfaces) et sur la gestion du point de vente à proprement : animer et motiver les équipes ; présenter et mettre en avant les produits ; gérer les stocks en magasin et dans les réserves ; organiser les services périphériques (service après-vente, garanties, livraison...), etc. Les compétences commerciales et marketing se sont considérablement développées dans les dix dernières années. Elles sont aujourd'hui très variées: connaître les marchés (concurrents, environnement législatif...) et les clients (modes de vie, besoins, comportements d'achat...) ; segmenter, cibler et différencier les offres ; définir l'assortiment, fixer et ajuster les prix en permanence ; gérer des opérations promotionnelles et les espaces publicitaires (parkings, radio, prospectus...).

L'importance de telle ressource ou de telle compétence dépend des facteurs clés de succès spécifiques du secteur considéré (alimentaire, bricolage, textile...) et du business model. Toutefois, dans une activité de commerce, la capacité à motiver les hommes est certainement une compétence clé. Cette compétence renvoie à la culture d'entreprise, aux principes de management, au recrutement, à la formation et aux systèmes de rémunération fixes et variables. Au fil du temps, on constate un élargissement des compétences nécessaires, l'aptitude à gérer des ressources intellectuelles (design, marques...) et relationnelles (partenaires, alliés...) se superposant aux compétences « cœur de métier » qui consistent à gérer des ressources physiques (stocks, surfaces de vente...). Les ressources relationnelles 
sont de plus en plus importantes, à l'instar de Starbuck (Gulati et Kleter, 2005), qu'il s'agisse des relations avec les clients, avec les fournisseurs, les partenaires ou les employés. Il en va de même pour la FNAC, qui réalise plusieurs milliers d'évènements chaque année dans ses magasins, en s'appuyant sur des relations étroites avec les producteurs de contenus (artistes, éditeurs...).

\section{L’offre et les activités réalisées par les distributeurs}

L'offre produits / services - En première analyse, l'offre de tout distributeur est composée d'un assortiment de marchandises mises à la vente. Cependant, tous les distributeurs n'ont pas la même stratégie d'assortiment. Par ailleurs, si la mise en vente d'un assortiment de produits est au cœur même de l'activité de commerce, la plupart des distributeurs ne se contentent pas de vendre des marchandises : l'offre de services s'est considérablement développée depuis quelques années (Lusch et al., 2007).

En ce qui concerne l'assortiment de marchandises, les distributeurs se distinguent par les univers de produits dans lesquels ils opèrent (alimentaire, équipement de la personne, aménagement de l'habitat, culture loisirs et sport...) et plus spécifiquement, par les familles de produits travaillées à l'intérieur de chaque univers (électroménager, meuble... à l'intérieur de «l'univers équipement du foyer »). Par ailleurs, au sein d'une même famille, on peut distinguer ceux qui développent plutôt l'assortiment en largeur (i.e., répondre à de nombreux besoins consommateur) ou en profondeur (i.e., multiplier les références pour chaque unité de besoin consommateur). Pour être encore plus spécifique, les stratégies d'assortiment valorisent plus ou moins les produits de fond de rayon (versus les produits saisonniers ou locaux), les marques nationales (versus les produits à marque de distributeur), etc. Bref, l'assortiment constitue un domaine dans lequel la liberté stratégique est forte, ces décisions ayant par ailleurs des conséquences très importantes sur les autres paramètres : coût d'achat des marchandises (plus faible quand le volume par référence augmente), marges arrières (plus élevées avec les marques nationales), surfaces de vente (croissante avec l'assortiment), etc. Le choix des formats de vente exploités est également un choix stratégique (hypermarchés, supermarchés, catalogues, sites marchands...), soit au niveau d'un groupe de distribution, soit au niveau d'une enseigne.

En plus d'un assortiment de marchandises, les distributeurs proposent une offre de services plus ou moins ambitieuse. Cette offre est composée de services de commodité destinés à minimiser les efforts transactionnels subis par le client (efforts, fatigue, temps, stress, risques) : horaires d'ouverture et emplacements de parking, information sur le produit, garderie pour les enfants, caisses rapides, garanties et extensions de garanties, reprises en cas de changement d'avis, livraison ou location de camionnettes, installation et mise en route de l'équipement, etc. Les distributeurs accompagnent l'acte d'achat d'un environnement de vente destiné à produire une expérience d'achat valorisée - en termes de distraction, d'évasion, de jeu ou simplement, de plaisir esthétique - et de nombreux services financiers (crédit gratuit, crédit à la consommation, voire produits d'épargne et d'assurance).

Le distributeur étant parfois un acteur social, l'offre de service ne concerne pas seulement des individus isolés mais des communautés (Lecher, 2002) : rencontres, animations, évènements et compétitions sportives ou culturelles, espaces à destination de la collectivité... Par ailleurs, les services ne concernent pas seulement les clients finaux mais également les fournisseurs (prestations logistiques, location d'espaces de vente ou d'espaces publicitaires...) voire même, les concurrents (location d'espaces dans les galeries marchandes et les centres commerciaux).

Les activités - Si l'achat-revente de produits constitue le cœur de métier d'un commerçant, la transformation de produits et/ou la réalisation de services sont des positions complémentaires à la revente. La simple intermédiation peut également être envisagée pour une partie de l'assortiment (le commerçant devient alors intermédiaire de commerce et se contente de mettre en relation l'acheteur et le vendeur). Cette question de la position dans un réseau de valeur est assurément stratégique et détermine en grande partie la nature même de l'entreprise et de ses activités. Elle doit être traitée au plus haut niveau et dépend en grande partie des ressources et compétences dont dispose l'entreprise. Il est probable que l'histoire personnelle des fondateurs - formation, expériences préalables, etc. - explique en grande partie la position prise dans le réseau de valeur.

L'entreprise ayant pris position dans un réseau de valeur, la question se pose ensuite de savoir quelles activités elle va réaliser elle-même, et quelles activités elle va déléguer à un prestataire, sous-traitant, partenaire ou allié. En effet, offrir tel produit ou tel service n'implique pas nécessairement d'en être (le seul) producteur. Les exemples sont nombreux de distributeurs qui proposent un service sans pour autant le délivrer eux-mêmes : quand un cuisiniste fait installer l'électro-ménager par un artisan, quand un distributeur de jouets fait emballer les articles par une association caritative, etc. Chaque activité dans la chaîne de valeur peut être ou non déléguée : conception, production, logistique, gestion du point de vente, management de la relation client, communication... Selon les ressources et 
compétences dont il dispose et selon les niveaux de revenus qu'il vise, le distributeur choisit de faire lui même ou de faire-faire. Il va de soi que faire (plutôt que déléguer) nécessite des ressources et des compétences spécifiques, mais constitue une source de revenus potentiels. La décision de développer un réseau en franchise - y compris au sein de groupes intégrés comme Carrefour (avec Shopi, Champion...) - s'inscrit aussi dans cette tendance au morcellement des chaînes de valeur. Il en va de même pour le recours à des partenaires à l'instar de Wal-Mart, leader mondial de la distribution, qui développe son assortiment de médicaments à bas prix et accole à ses magasins des centres médicaux gérés par des prestataires spécifiques.

L'externalisation touche aussi le secteur du commerce, notamment la vente à distance. Une étude récente menée par la FEVAD (Fédération du e-commerce et de la vente à distance) montre que les emplois indirects dans ce secteur sont substantiels, relativement aux emplois directs (respectivement 30000 contre 50 000). L'analyse détaillée montre qu'il s'agit essentiellement de prestations de logistique (20 000 emplois) et de centres d'appels (7 000 emplois).

En matière de commerce, les partenariats sont fréquents. Dans certains cas, ils peuvent induire une prise de participation capitalistique. Les partenariats commerciaux peuvent être équilibrés par des actions qui se compensent dans les deux sens (commercialisation des produits de l'un sur les espaces de l'autre...), par un apport d'espaces publicitaires (site, newsletter...) et d'espace de vente ou encore, par des paiements (commissions sur les ventes réalisées). Ainsi, Rue du Commerce met en ligne sur le site de France Loisirs une boutique de produits de haute technologie, pendant que France Loisirs met en ligne sur le site de Rue du Commerce des produits audio à télécharger. Rue du Commerce va également ouvrir une dizaine de corners (espaces de vente) dans les boutiques de France Loisirs (sur les 200 que possède l'enseigne). Le partenariat peut évoluer vers l'élargissement à de nouvelles catégories de produits, ou le retrait dans les boutiques France Loisirs des produits achetés sur le site Rue du Commerce.

\section{Les revenus générés et les charges subies par les distributeurs}

Les sources de revenus - Les BM des distributeurs s'appuient essentiellement sur la revente de marchandises, qui représente plus de 90\% de leur chiffre d'affaires (INSEE, 2004). Toutefois, d'autres sources de revenus périphériques se développent à partir des ressources et compétences développées. Les revenus liés à la revente de produits sont au cœur des activités des distributeurs: la marge commerciale sur les produits vendus, qui correspond à la différence entre la vente de marchandises et le coût d'achat de ces mêmes marchandises, est communément de $40 \%$ dans le commerce spécialisé (équipement de foyer, produits culturels, etc.). Elle est généralement plus faible dans le commerce alimentaire (entre 13 et 20\%). Toutefois, cette marge est compensée par des avantages financiers dans la mesure où les marchandises sont vendues avant que les fournisseurs ne soient payés : le besoin en fond de roulement étant structurellement négatif, l'activité génère des produits financiers importants. Les distributeurs ont par ailleurs développé des marques propres (dites marques de distributeurs ou MDD) qui leur permettent de cumuler différents niveaux de marge (marge de distribution et une partie de la marge de l'industriel).

La marge commerciale sur les produits vendus (que l'on appelle aussi marge avant) est bien souvent complétée par des remises conditionnelles (remise sur volume, par exemple) et par la vente de prestations (ou coopération commerciale) qui forment ce que l'on appelle les marges arrières. Certaines, notamment les coopérations commerciales, interviennent après la signature du contrat de vente et ne sont généralement pas connues au moment de signer le contrat. Par exemple, quand un marchand propose à une marque un espace spécifique sur son point de vente (tête de gondole, par exemple) ou la mise en avant d'une nouveauté, le distributeur obtient un financement spécifique de la part de l'industriel. Les marges arrière peuvent fréquemment représenter $15 \%$ à $20 \%$ du prix de vente final au client. C'est donc aujourd'hui une source de revenus importante, qui est cependant destinée à disparaître progressivement, le législateur s'étant emparé de cette question (circulaire Dutreil de 2005 réformant la loi Galland de 1996, suivie de modifications législatives attendues pour 2008).

Le total des marges issues de la vente de produit est appelé la marge brute. C’est la différence entre le chiffre d'affaires incluant les marges arrière et le coût d'achat des marchandises vendues. Cependant, en prolongement d'une conception stricte du commerce (qui consiste à revendre des produits en l'état), la revente de services associés au produit rentre dans la sphère du commerce. Par exemple, quand un commerçant vend une garantie complémentaire pour l'achat d'un ordinateur, ou quand il vend une assurance contre le vol pour l'achat d'un téléphone mobile, il s'agit d'un service directement lié à l'achat d'un produit (on ne peut pas vendre la garantie ou l'assurance sans vendre le produit).

Le distributeur peut également percevoir d'autres revenus périphériques liés à la vente de marchandise, à savoir les cotisations mensuelles ou annuelles que les clients sont parfois obligés de payer pour avoir accès au magasin. Par exemple, les cotisations d'adhésion s'élèvent chez Costo à 45 dollars par an et 100 dollars pour une adhésion « client privilégié » 
qui permet d'avoir accès à des services et promotions additionnels. Ces cotisations représentent environ $2 \%$ des revenus des warehouse clubs (Kim et Choi, 2007), sachant qu'il s'agit quasiment d'une marge nette dans la mesure où les charges liées à ces revenus sont quasiment nulles. Au delà de cette source additionnelle de revenus, les cotisations d'abonnement présentent un avantage marketing non négligeable car cela permet à l'enseigne de fidéliser les clients et de se concentrer sur les plus gros clients et ainsi de maximiser la profitabilité de l'enseigne (Kim et Choi 2007).

Pour générer un revenu lié à la vente des marchandises, le distributeur n’est pas obligé de posséder un stock. Il peut cantonner son rôle à une simple intermédiation entre le client final et une autre entreprise ou entre les clients eux-mêmes. Le business model n'est plus transactionnel mais relationnel. Le distributeur se contente de mettre en relation les deux parties sans concevoir, produire ou livrer. Les marchandises sont alors en dépôt vente et le distributeur entrepose les marchandises, éventuellement conseille les acheteurs et assure l'intermédiation financière. La commission peut être variable (selon le montant de la transaction) ou fixe.

Les distributeurs peuvent s'appuyer sur leurs ressources et compétences pour dégager de nouvelles sources de revenus auprès des industriels (commercialisation des données de vente en partenariat avec des panélistes, vente d'espaces commerciaux...) et auprès d'autres distributeurs (commercialisation d'espaces de vente dans les galeries marchandes, redevances pour concessions, licences, marques commissions...). La gestion immobilière se sophistique et la valorisation des espaces peut faire naître des sources de revenus originales, comme la production d'énergie sur le toit des points de vente.

On peut très bien envisager qu'à l'avenir, les distributeurs travaillent davantage les autres sources de revenus, à l'image des acteurs du commerce électronique. Par exemple, Amazon développe fortement ses prestations logistiques pour le compte d'autres sites de commerce électronique... au point que certains experts s'interrogent sur le fait de savoir si Amazon est bien encore un cyber-marchand. Les acteurs du commerce électronique ayant été amenés à développer des technologies assez pointues il est assez naturel de les voir revendre ces technologies à des clients... ou même à des concurrents. Les leaders y voient une possibilité de croissance intéressante et, plus stratégiquement, une façon de contrôler indirectement leurs concurrents. Il en va ainsi de eBay, qui licencie sa technologie Paypal, et fait de lui un acteur (bientôt majeur) des services financiers.
L'équilibre des revenus et des charges - A un flux de revenus correspond un flux de charges et l'objectif consiste naturellement à maximiser la différence, au moins sur le moyen terme.

Le niveau de marge et la structure des coûts varient fortement d'un distributeur à l'autre. Les taux de marge et les structures de coûts sont très variables selon les types de surfaces de vente. Plus la marge est faible, plus la structure de coûts doit être légère (tableau 1).

Tableau 1 - Taux de marge et structure des coûts d'exploitation

\begin{tabular}{|l|l|l|l|}
\hline & $\begin{array}{l}\text { Hard discount } \\
\text { (Lidl, Netto...) }\end{array}$ & $\begin{array}{l}\text { GSA } \\
\text { (Carrefour, Auchan...) }\end{array}$ & $\begin{array}{l}\text { Grands magasins } \\
\text { (Galeries Lafayette, Printemps...) }\end{array}$ \\
\hline Marge & 12 à $14 \%$ & 18 à $22 \%$ & 35 à $40 \%$ \\
\hline Frais de personnel & $1 / 3$ de la marge & $1 / 2$ de la marge & 2/3 de la marge \\
\hline
\end{tabular}

Les marges peuvent également varier de façon très importante selon les catégories de produit, sachant que l'objectif de la politique de prix est de maximiser la rentabilité globale de l'ensemble de l'assortiment. Elle ne doit pas être appréciée au niveau de chaque référence mais au niveau de l'ensemble de la catégorie (Ducrocq, 2006). Ainsi, le distributeur peut être amené à proposer des services gratuits et réaliser une marge très faible, voire vendre un certain nombre de produits à prix coûtant. Ces arbitrages doivent prendre en compte le positionnement de l'enseigne, l'analyse des fonctions des produits, la sensibilité des clients à certaines catégories de produit, les actions promotionnelles des concurrents, etc.

Politique de prix - Les distributeurs peuvent soit choisir de proposer un nombre de produits à prix cassé pendant de courtes périodes de temps (politique HI-LO : High-Low prices) soit privilégier une politique du prix bas permanent, quelle que soit la période de l'année (politique EDLP : Every Day Low Prices).

La technique des offres temporaires présente plusieurs avantages (Berman et Evans, 2004) : elle permet davantage de flexibilité (par rapport au choix des produits mis en avant et au choix des cibles) ; les campagnes promotionnelles sont souvent payées (en partie ou totalement) par les industriels ; elles permettent de créer du trafic vers le point de vente et de recruter de nouveaux clients ; elles donnent pleine satisfaction au consommateur qui a l'impression de faire de bonnes affaires (et par voie de conséquence, d'augmenter le panier d'achat). Mais, la mise en place des promotions demande une organisation interne importante (choix des produits mis en avant, négociation avec les industriels, communication nationale et/ou locale, implantation dans les linéaires...) et produit des effets seulement à court terme. 
Elle modifie peu les comportements de choix de point de vente dans le long terme (Volle, 1999).

En revanche, la politique du prix le plus bas (EDLP) permet d'économiser tous les surcoûts engendrés par les opérations promotionnelles. Elle permet au distributeur de se concentrer sur des activités de fond et donne au consommateur l'impression d'équité et d'honnêteté (Filser et al, 2001). Toutefois, cette politique est difficile à mettre en œuvre et n'est pas forcement adaptée à toutes les formes de commerce. Dans la lignée de Wal-Mart et d'Ikéa, de nombreux distributeurs ont essayé de la mettre en place mais, beaucoup ont dû faire marche arrière. Seuls, quelques discounters ont réussi dans cette voie. En effet, cette stratégie conduisant les distributeurs à rogner significativement sur leur marge, ils sont obligés de compenser par une plus grande rotation des stocks sur un assortiment limité, ce qui correspond au modèle économique des discounters (Dion et Sueur, 2006). Par ailleurs, cette politique ne convient pas aux marchés sur lesquels la demande et/ou l'offre est fluctuante car le distributeur est obligé de faire varier ses prix en fonction de la raréfaction des ressources à certaines périodes de l’année comme les fruits et légumes, par exemple (Berman et Evans, 2004).

Le choix entre EDLP et HI-LO dépend des caractéristiques du secteur (périssabilité et hétérogénéité de l'assortiment), des caractéristiques de l'entreprise (nombre de magasins, différenciation, taille des magasins), des concurrents (stratégies de promotion et de prix) et des caractéristiques des consommateurs (Voss et Seiders, 2003).

La distinction entre sources de revenus et sources de profit - En matière de business model, il est essentiel de bien distinguer les sources de revenus et les sources de profit. Les revenus annexes peuvent peser faiblement dans le chiffre d'affaires mais représenter une part très importante du profit dans la mesure où les taux de marge sont nettement supérieurs. Si le résultat net de Carrefour représente 3\% du chiffre d'affaires, celui de Google en représente $30 \%$ : en l'occurrence, le modèle de revenu publicitaire dégage une marge nette sensiblement supérieure à celui de l'achat/revente. De fait, la vente de prestations ou les revenus publicitaires peuvent représenter 5\% du chiffre d'affaires, mais 50\% des profits. Il est donc essentiel d'allumer ces autres moteurs de croissance pour atteindre plus sûrement ou plus rapidement la zone de profitabilité. C'est sans doute ce mécanisme qui a poussé les commerçants électronique à diversifier leurs sources de revenus, relativement aux commerçants traditionnels dont le modèle repose essentiellement sur la revente de marchandises.
Le besoin capitalistique (investissement nécessaire pour ouvrir un magasin) est aussi un élément discriminant. Certains distributeurs ont besoin d'un chiffre d'affaires minimum par magasin. D'autres sont très rentables avec des chiffres d'affaires faibles ce qui nécessite un coût d'entrée et une structure plus légère. Par exemple, Castorama et Brico-Dépôt, tout en ayant le même actionnaire, ont des business models très différents.

\section{2 - Les chemins de la performance dans la distribution}

Les sources de revenus diffèrent et chaque source peut être configurée très différemment d’un distributeur à l'autre. Par exemple, le revenu issu de la revente de marchandise n'est pas du tout travaillé de la même façon par les distributeurs traditionnels et par les discounters (moindre profondeur de l'assortiment, politique de prix bas permanents, faible communication...). L'examen des stratégies menées par les distributeurs montre que deux grands BM co-existent dans le secteur du commerce (e.g., Benoun et Heliès-Hassid, 2003 ; Waldman et Thoenig, 2005 ; Lusch, Vargo et O’Brien, 2007)

Le premier business model (BM-X) consiste à simplifier la proposition de valeur, tout en diminuant les coûts pour gagner en efficience. Le second business model (BM-Y) consiste à enrichir la proposition de valeur pour gagner en attractivité « par le haut », en traduisant dans l'organisation l'hétérogénéité des préférences des consommateurs. Les entreprises caractéristiques du BM-X sont, par exemple Aldi, Wal-Mart, Conforama. Les entreprises du sont BM-Y, par exemple Monoprix, Auchan, Darty, Truffaut ou la FNAC.

L'analyse que nous proposons ne recouvre pas exactement la distinction classique entre domination par les coûts et différenciation dans la mesure où chaque business model peut induire un degré de différenciation fort. Par ailleurs, elle présente quelques avantages par rapport aux visions classiques en usage dans le secteur du commerce. De fait, traditionnellement, la diversité au sein du secteur de la distribution est appréhendée à travers les univers de produits (l'alimentaire, le bricolage...) et les formats (hypermarchés, grands magasins, vente par catalogue....). Ici, notre propos consiste à dire que dans chaque univers et au sein de chaque format, on peut retrouver des entreprises qui incarnent le BM-X ou qui incarnent le BM-Y. Ainsi, dans le secteur du bricolage, Brico-Dépôt incarne le BM-X alors que Leroy Merlin incarne le BM-Y. Pour le format des supermarchés, Monoprix incarne le BM-X et Lidl incarne le BM-Y. L'approche par les business models est donc une alternative aux approches traditionnelles employées dans le secteur, par l'INSEE notamment. 
Le niveau de marge nette (revenus - charges) ne constitue pas une dimension très discriminante, car les deux business models peuvent être également profitables. Ce qui distingue ces BM ce sont plutôt les processus par lesquels la marge est construite et plus précisément, le fait de savoir si la création de valeur pour le client passe par une simplification ou par un enrichissement de la proposition de valeur.

Pour caractériser plus précisément ces business models à partir des éléments constitutifs présentés dans la première partie, on peut dire que le management des ressources et des compétences est probablement la dimension la plus discriminante. Ceci dit, comme le montre le tableau 1, de nombreux éléments diffèrent, notamment les sources de revenus.

\begin{tabular}{|c|c|c|}
\hline & BM-X & BM-Y \\
\hline Ressources & $\begin{array}{l}\text { Essentiellement physiques et } \\
\text { logistiques }\end{array}$ & $\begin{array}{l}\text { Plus de ressources symboliques } \\
\text { (marques...) et relationnelles }\end{array}$ \\
\hline Compétences & $\begin{array}{l}\text { Optimisation des processus et } \\
\text { capacité à simplifier la proposition } \\
\text { de façon pertinente }\end{array}$ & $\begin{array}{l}\text { Innovations destinées à enrichir la } \\
\text { proposition de valeur }\end{array}$ \\
\hline Offre & $\begin{array}{l}\text { Limitée (en largeur et surtout, en } \\
\text { profondeur), peu de services }\end{array}$ & $\begin{array}{l}\text { Assortiment large et profond, } \\
\text { services complémentaires }\end{array}$ \\
\hline Activités & $\begin{array}{l}\text { Tendance à l'intégration, peu } \\
\text { d'enseignes / canaux, voire un seul }\end{array}$ & $\begin{array}{l}\text { Tendance à l'externalisation, } \\
\text { nombreuses enseignes et multi-canal }\end{array}$ \\
\hline Revenus & $\begin{array}{l}\text { Essentiellement la marge } \\
\text { commerciale }\end{array}$ & $\begin{array}{l}\text { Marge brute (incluant les marges } \\
\text { arrière) et divers autres revenus }\end{array}$ \\
\hline Marge & $\begin{array}{l}\text { Marge commerciale plus faible, } \\
\text { mais forte rotation des stocks ( } \rightarrow \\
\text { marge totale élevée), politique de } \\
\text { prix bas permanents (EDLP) }\end{array}$ & $\begin{array}{l}\text { Egale, voire plus faible, politique } \\
\text { promotionnelle active (HI-LO) }\end{array}$ \\
\hline
\end{tabular}

A titre d'exemple, la comparaison systématique entre Borders et Barnes \& Noble montre que le premier met l'accent sur la profondeur de l'assortiment (et les systèmes d'information pour gérer la complexité associée à un assortiment profond) alors que le second met l'accent sur les prix et les volumes par référence, le 20/80 (Raff, 2000). Les postures adoptées sont le reflet de l'histoire des deux entreprises, Border ayant une compétence forte en bases de données dans le secteur de la littérature.

Cependant, ces deux business models doivent être envisagés comme des idéaux-types. Il est rare qu'une même entreprise n'emprunte pas, d'une façon ou d'une autre, aux deux chemins de la performance (simplifier la proposition de valeur et optimiser les ressources \& compétences pour gagner en efficience / enrichir la proposition de valeur pour gagner en attractivité), à l'instar de Zara ou d'IKEA. C'est d'ailleurs la raison pour laquelle nous avons dénommés ces business models « $\mathrm{X}$ » et " $\mathrm{Y}$ », laissant entendre qu'ils constituent des continuums, et peuvent être analysés comme les axes d'un espace stratégique à deux dimensions (abscisse et ordonnée).

\section{BM-X : Optimiser les ressources et compétences pour gagner en efficience}

Dans cette logique, les efforts sont concentrés sur l'amélioration de la performance des processus (achats, livraison, rotation des stocks...). L'objectif est de rationaliser l'utilisation des ressources et des compétences pour rendre les processus plus efficients. Pour cela, les distributeurs peuvent orienter leurs efforts vers quatre objectifs : l'allègement ou le report des coûts, le développement d'économies d'échelle, l'accélération de la rotation des stocks et la fluidification les échanges entre les partenaires du canal de distribution (supply chain).

Alléger les coûts d'exploitation - Il s'agit de fonctionner en consommant le moins de ressources possibles. L'objectif est de faire des économies à tous les niveaux : coûts d'achat des marchandises, coûts d'exploitation des magasins, investissements immobiliers, etc. Toutefois, alléger les coûts d'exploitation ne signifie pas forcement diminuer les dépenses. Cela passe également par l'innovation technologique pour faciliter le travail sur le point de vente et dans les entrepôts, mais aussi pour optimiser le transport des marchandises et la circulation des flux d'information. Il peut s'agir d'innovations technologiques (codes barres, puces RFID, étiquettes électroniques, caisses automatiques, applications informatiques pour gérer les stocks, réassorts automatiques...) mais aussi d'innovations marketing (organisation du point de vente, packaging, information sur le lieu de vente...). Ces innovations sont vitales pour les distributeurs qui se battent dans le cœur de marché afin de réduire les coûts d'exploitation et d'améliorer simultanément le service client (Sethuraman et Parasuraman, 2005).

Toutefois, économiser trop sur les coûts d'exploitation n'est pas forcement la meilleure stratégie à court et moyen terme. Comme le démontre Cascio (2006) à partir d'une analyse comparative des coûts de personnel chez Sam's Club (groupe Wal-Mart) et Costco, deux discounters américains. Chez Costco, les salaires sont bien supérieurs à ceux de Sam's Club (en moyenne 17 dollars par heure, contre 10,1 chez son concurrent). Les frais de personnel représentent $70 \%$ des coûts d'exploitation, soit $40 \%$ de plus que Sam's Club. Cependant, il est important d'adopter une vision plus large de la structure des coûts pour intégrer l'ensemble des activités et des centres de coûts de l'entreprise. Ainsi, chez Costo, le 
turnover est très faible (6\% à l'issue de la première année), les salariés sont plus compétents et leur productivité, mesurée en chiffre d'affaires par employé, est sensiblement plus élevée. Certes, les salaires sont $40 \%$ plus élevés que chez Sam's Club mais au final, les coûts de main d'œuvre sont plus faibles (5,55 \% chez Costco et 6,25 \% chez Sam's Club). Chez Sam's Club, le faible niveau des salaires se traduit par un turnover très important, des conflits et une relation-client dégradée.

Au-delà des économies sur les coûts d'exploitation, certains distributeurs ont poussé la démarche plus en amont pour s'intéresser aux coûts de production des marchandises vendues. Wal-Mart, par exemple, a forcé la porte de nombreuses usines pour faire analyser, par des contrôleurs de gestion de l'enseigne, la décomposition des coûts de production de nombreux produits de grands groupes industriels (Procter, Unilever...).

Externaliser les coûts - Economiser suppose parfois de reporter les coûts sur les partenaires (clients, fournisseurs, collectivités). Par exemple, le distributeur peut associer le client au processus de production et de mise à disposition de l'offre. Moyennant des prix beaucoup plus faibles, les clients d'IKEA acceptent de parcourir de longues distances pour se rendre en magasin, de monter leurs meubles eux-mêmes, d'installer leurs équipements électroménager... Plutôt que de facturer ces prestations aux clients, IKEA préfère proposer aux clients de les prendre en charge eux-mêmes. Des coûts peuvent également être externalisés vers les fournisseurs, à travers la facturation de coopérations commerciales (réalisation des prospectus promotionnels, animateurs commerciaux en magasin, etc.).

L'externalisation peut parfois concerner l'ensemble de la collectivité. Il en va ainsi de WalMart qui a reporté sur les Etats la prise en charge de la protection sociale de ses salariés. Les salariés étant mal payés et leur protection sociale peu enviable, ce sont souvent les Etats qui sont contraints d'accompagner socialement les salariés. Le Demographic Staff of the Commitee on Education and the Workforce estime qu'un magasin Wal-Mart de 200 personnes coûte à l'Etat Fédéral 420750 dollars par an, soit 2103 dollars par employé (Cascio, 2006).

Générer des économies d'échelle sur le retailing mix - Il est possible de générer des économies d'échelle à partir d'une standardisation des points de vente. Le développement en franchise en est un bon exemple. En créant des points de vente standardisés avec un design type, des plans merchandising et des assortiments standards, les distributeurs peuvent limiter les coûts d'exploitation des magasins et ouvrir à moindre coût de nouveaux points de vente. Mais, il n’est pas toujours facile de réaliser des économies d'échelle car certains éléments du retailing mix s'y prêtent plus difficilement. Par exemple, l'ambiance du point de vente est très difficile à dupliquer (Barber et Tietje, 2004). En fonction de l'affluence, la façon dont les clients perçoivent le point de vente risque d'être très différente (Dion, 1999). Par ailleurs, s'il est possible de standardiser l'environnement d'achat (matériaux et design, musique, tenues vestimentaires....), l'orientation client du personnel en contact ne prête moins facilement à ces démarches de normalisation. S'il est possible de former le personnel à l'accueil client, la sincérité du sourire et l'authenticité de l'engagement commercial ne se commande pas...

Une autre façon de développer des économies d'échelle passe par la standardisation des compétences. Toutefois, une compétence sur un marché n’est pas forcement transférable sur un autre. Par exemple, lors des premières étapes de son internationalisation, Carrefour avait choisi de dupliquer in-extenso son savoir-faire (format des magasins, procédures de gestion des points de vente et modes de management). Toutefois, le groupe s'est rapidement aperçu qu'il était important d'adapter non seulement l'offre mais aussi les modes de management. Par exemple, en Pologne, on a pu constater une phase d'adaptation réciproque qui a permis une évolution des compétences et notamment des modes de management en fonction de l'héritage culturel de chacun, à la fois des managers français et polonais de la filiale polonaise et des opérationnels polonais (Hurt et Hurt, 2005).

Par ailleurs, la recherche d'économies d'échelle n'est pas forcement une nécessité (Barber et Tietje, 2004). Ainsi, un petit distributeur local de produits de bricolage ne peut développer des économies d'échelle comme peut le faire par exemple Home Depot, leader mondial du secteur. Il doit alors mettre en avant d'autres ressources et compétences, par exemple, le conseil, la proximité ou la qualité de la relation client.

Accélérer la rotation des stocks - Les niveaux de stock sont très variables selon les enseignes, et ces écarts ont naturellement un impact considérable sur la trésorerie. Le besoin de couverture de stock dépend de nombreux facteurs : la proximité des fournisseurs (plus ils sont loin, plus il faut commander en avance), le risque de rupture sur le produit (prévisibilité des ventes et capacité du fournisseur à réassortir), la taille des assortiments (plus les assortiments sont étroits, plus les stocks tournent vite) et évidemment, en toile de fond, l'excellence opérationnelle du distributeur. Cette capacité à faire tourner les stocks rapidement est au cœur du fonctionnement des modèles de discount (cf. tableau 3).

C’est dans cet objectif que Wal-Mart a développé le category management, voilà une dizaine d’années. L’objectif consistait à coopérer avec les industriels pour identifier les références clé 
de chaque catégorie, exclure les références qui n’apportent pas de réelle valeur au consommateur et ainsi conserver dans l'assortiment un nombre limité de références avec un taux de rotation plus élevé.

Tableau 3 - Comparaison du hard et soft discount (Benoun et Heliès-Hassid, 2003)

\begin{tabular}{|l|c|c|}
\hline & Hard discount & Soft discount \\
\hline Prix de vente & 80 & 100 \\
\hline Prix d'achat & 68 & 70 \\
\hline Marge & 12 & 30 \\
\hline Nombre de rotations des stocks par an & 12 & 4 \\
\hline Chiffre d'affaires & 960 & 400 \\
\hline Marge globale & 144 & 120 \\
\hline
\end{tabular}

La rotation des stocks est un élément stratégique pour de nombreux distributeurs. Par exemple, Zara dispose d'un outil de production très flexible proche de sa clientèle et un système d'information intégré à toute la filière qui permet de produire des séries très courtes (de 10 à 15000 exemplaires). Ce système de production présente un certain nombre davantage à la fois sur le plan de l'efficience opérationnelle mais aussi sur le plan de l'efficacité marketing : suppression des stocks, limitation des risques de produire une collection qui ne plait pas au client, adaptation continue des collections en fonction des goûts des clients et des collections des concurrents, création de la rareté (le renouvellement permanent de l'offre pousse le client à revenir régulièrement pour découvrir les nouveautés et à acheter dès qu'il trouve un produit qui lui plaît de peur qu'il ne soit plus là dans les jours voir même les heures à venir).

Fluidifier les échanges entre les partenaires du canal de distribution - Les distributeurs se sont engagés dans une rationalisation des approvisionnements : centralisation des informations et des flux, suppression des stocks en magasin, développement de plateformes régionales ou nationales, réassorts automatisés et mise en place d'outils d'échange d'informations comme l'EDI (Echange de Données Informatisées), la GPA (Gestion Partagée des Approvisionnements) ou le CPFR (Collaborative Planning, Forecasting and Replenishment, gestion concertée des prévisions des ventes). Cette optimisation de la chaîne logistique nécessite une collaboration étroite avec tous les acteurs du canal (industriels, transporteurs, points de vente, entrepôts...). Par exemple, la FNAC et Samsung ont mis en place une gestion partagée des approvisionnements pour un certain nombre de références. La FNAC transmet au constructeur des données sur les encours, les stocks, les marchandises livrées en magasin et les marchandises éventuellement manquantes lors des précédentes livraisons (Dion et Sueur, 2006). A partir de ces informations, Samsung calcule les besoins de l'enseigne et transmet au distributeur une proposition de commande qui tient compte du stock de sécurité souhaité, du mode de palettisation désiré et du planning de livraison du distributeur. La FNAC doit ensuite procéder à une simple validation des propositions de réapprovisionnement du producteur. La marchandise est livrée trois jours plus tard. La GPA permet au distributeur de lisser les charges, de mieux gérer la réception des marchandises (anticipation des livraisons : date, heure et volumes), d'éviter les complications administratives et de baisser le niveau du stock de sécurité de 18 à 15 jours. La mise en place de livraisons régulières a permis à Samsung de lisser sa production, d'optimiser ses livraisons et d'éliminer les surcoûts de livraison liés aux commandes incomplètes.

Parfois, les partenariats logistiques peuvent inclure plusieurs industriels en parallèle. Carrefour a, ainsi, mis en place un pooling industriel avec Benedicta et Nutrimaine (Banania et Benco). Les deux industriels coopèrent pour mutualiser les flux physiques (entreposage et transport) et les flux informatiques à partir d'une plate-forme logistique unique. Les 15 entrepôts Carrefour disposent ainsi d'un interlocuteur unique concernant l'approvisionnement de ces trois marques (avec une livraison groupée et une proposition de commande unique). Carrefour envisage d'intégrer d'autres industriels dans le processus (Dion et Sueur, 2006).

Le développement de partenariats entre fournisseurs et distributeurs au niveau de l'assortiment et de la logistique nécessite une évolution des relations industriels-fournisseurs. Ces nouvelles formes de coopérations permettent aujourd'hui de ne plus envisager les relations fournisseur-distributeur comme un rapport de force mais comme une relation gagnant-gagnant permettant d'anticiper et de réagir rapidement aux fluctuations de la demande. Ainsi, le distributeur devient le pivot du canal de distribution qui permet l'interface entre les industriels et les consommateurs finaux et non plus un écran entre les industriels et le marché. La performance du distributeur provient donc aussi de sa capacité à fluidifier les échanges au sein du canal de distribution pour permettre une bonne circulation des flux de marchandises et d'information. Ainsi, grâce à une chaîne d'approvisionnement extrêmement performante qui s'appuie sur des façonniers essentiellement basés en Espagne et au Maghreb et une logistique intégrée, Zara n’a besoin que de trois semaines pour mettre un produit sur le marché contre deux à trois mois pour son concurrent H\&M (soit des délais de mise sur le marché 12 fois plus court). Une commande reçue d'un magasin en Europe est livrée sous 24h et $48 \mathrm{~h}$ pour un magasin localisé en Asie ou au Etats-Unis. 


\section{BM-Y : Enrichir la proposition de valeur pour gagner en attractivité}

Dans cette deuxième approche, le distributeur ne recherche pas spécifiquement l'efficience mais l'attractivité de sa proposition de valeur. Il cherche à créer de la marge non pas à partir de la rationalisation des ressources et compétences mais à partir de l'offre (e.g., Rieunier et Volle, 2002 ; Badot et Benoun, 2005). L’objectif est de créer de la valeur à partir de la connaissance client, en intégrant le client dans le processus de production de l'offre, en faisant converger les canaux de distribution vers le client et en développant des services associés.

Exploiter la connaissance client - Par rapport à l'industriel, le distributeur bénéficie d'un avantage majeur : la proximité avec le client. Alors que les industriels sont souvent obligés de mettre en place des protocoles d'étude complexes et fastidieux pour connaitre leurs clients, les distributeurs ont le grand privilège d'être en contact direct et quotidien avec les consommateurs. Cette proximité leur permet de développer une connaissance client très fine. Par exemple, chez Matalan ${ }^{3}$, distributeur britannique de prêt-à-porter et de décoration discount, seuls les clients munis de la carte de l'enseigne ont la possibilité de réaliser des achats. Mais contrairement aux clubs entrepôts américains (warehouse clubs), la carte est gratuite. L'objectif n'est pas de s'assurer une source de revenus supplémentaire ou de fidéliser les clients mais de générer de la connaissance client. En 2004, l'enseigne disposait d'une base de données de 10 millions de clients. Grâce à ce système, l'enseigne connait parfaitement sa clientèle (profil socio-démographique, motivations de visite, paniers d'achats réalisés, fréquence de visite, sensibilité aux promotions...). L’enseigne est également capable de réaliser une analyse très fine des ventes (par catégorie de produit, par référence, par marque, par zone géographique et par magasin). A partir de cette connaissance, l'enseigne peut optimiser les assortiments au niveau de chaque point de vente afin que chacun possède l'assortiment qui correspond le mieux au profil et attentes de la zone de chalandise et mettre en place des opérations de marketing direct très ciblées (Rowley et Haynes, 2005).

Cette connaissance confère une meilleure capacité d'adaptation et d'innovation dans des environnements dynamiques et complexes (Bolton et al., 2007). Par exemple, chez Zara, la création de nouveaux modèles est issue de deux éléments : les tendances de la mode (dictée essentiellement par les grands couturiers) et le système d'information qui permet une traçabilité très fine des ventes (par tissu, par couleur, par type et style des pièces vendues et

${ }^{3}$ L'enseigne crée en 1983 compte, fin 2007, 190 magasins en Grande-Bretagne et Ecosse. par point de vente). Les stylistes créent de nouveaux modèles qui répondent aux mieux aux attentes des clients avec un ciblage très fin puisque les assortiments de chaque magasin sont ajustés en permanence en fonction des données de sortie de caisse de chaque point de vente. Ainsi, en 2005, plus de 30000 modèles différents sont sortis des bureaux d'Inditex (maison mère de l'enseigne).

Créer de la valeur pour le client - Distribuer un produit, ce n’est pas simplement mettre à disposition du client le «bon produit au bon endroit, au bon moment ». C'est proposer une combinaison de produits, de services et d'informations. Il ne s'agit pas de d'acheter des produits pour les revendre, mais de vendre des solutions à valeur ajoutée (Gulati et Kletter, 2005). Dans cette logique, Truffaut ne vend pas des plants à repiquer mais l'opportunité d'embellir son cadre de vie. De la même façon, Starbuck ne vend pas des tasses de café mais une expérience en situation de mobilité urbaine.

Créer de la valeur ne signifie pas forcement vendre plus cher. L'important, c'est d'offrir la meilleure valeur pour le client au meilleur prix. Pour créer de la valeur, il faut également prendre en considération la chaîne de valeur inversée, c'est-à-dire la seconde vie des produits : réparation des produits défectueux, enlèvement et recyclage des produits hors d'usage et des produits abimés, revente des produits d'occasion, valorisation des déchets... La seconde vie du produit ne doit pas être considérée comme un coût mais comme un avantage compétitif concurrentiel (Jayaraman et Luo, 2007). Par exemple, Décathlon a su saisir cette opportunité pour créer du trafic vers ses magasins et multiplier les occasions d'achat à travers le développement de la Trocathlon. Dans cette opération, tout le monde est gagnant: les vendeurs qui n’ont pas à se soucier de la revente de leur matériel usagé, qui se limitent à le déposer chez Décathlon et reçoivent ensuite par la poste un bon d'achat équivalent à l’intégralité du prix de vente; les acheteurs qui ont l'opportunité d'acheter un matériel d'occasion en bon état et avec le conseil des vendeurs ; le distributeur qui créée du trafic sur le point de vente et multiplie les occasions d'achat. Ce faisant, le distributeur n'est plus un simple intermédiaire qui achète et revend des marchandises mais un intermédiaire de commerce qui met en relation des acheteurs et des vendeurs, au même titre qu'Ebay ou Priceminister.

Le distributeur ne doit pas simplement se limiter à créer de la valeur pour le consommateur final mais également pour la collectivité au sens large. Il est un acteur de la vie locale, doit assumer ce rôle et exploiter les opportunités générées par cette proximité. En donnant 
systématiquement la priorité au recrutement local et aux filières d'approvisionnement locales, les distributeurs dynamisent le tissu économique et l'image de l'enseigne. Ils deviennent des partenaires pour co-créer de la valeur avec les acteurs du tissu économique local (Lusch et al. 2007). Ainsi, chez Carrefour, plus de 90\% des produits mis en rayon proviennent de producteurs et de fabricants nationaux, un chiffre sensiblement plus bas chez Wal-Mart (autour de 50\%).

Coproduire de la valeur avec le client - Le consommateur ne doit plus être considéré comme externe à l'entreprise. A l'heure où les frontières organisationnelles deviennent poreuses, avec la notion d'entreprise étendue et de réseau, il doit être intégré au processus de création de valeur. Le client est une ressource capable de générer de nouvelles ressources, un partenaire capable de co-créer de la valeur avec l'entreprise pour produire une valeur et l'entretenir dans le temps (Lush et al., 2007). Il ne s'agit plus simplement de commercialiser des produits pour mais avec un client. Dans cette logique, la FNAC - Eveil \& Jeux associe les parents dans le processus de sélection de l'assortiment.

Toutefois, la co-création va bien au delà, dans la mesure où le point de vente n'est plus aujourd'hui un simple lieu de stockage et d'exposition, mais un support d'expériences (e.g., Hetzel, 2002). De nombreux individus fréquentent des points de vente pour se distraire, sortir de leurs routines quotidiennes, vivre des expériences différentes.... Le point de vente devient un lieu de récréation, d'amusement, d'expériences et de mémoire (retail-entertainement), un lieu au sein duquel les consommateurs peuvent ré-enchanter leur quotidien en découvrant des objets nouveaux ou insolites, en participant à des activités différentes... On va faire rêver les clients devant des prototypes (Atelier Renault sur les Champs Elysées) ou pseudo-prototypes (collection « défilé » dans les magasins de prêts à porter Kenzo). On donne la possibilité aux clients d'exprimer leurs compétences (possibilité de grapher les murs et d'utiliser les rampes de skates et de roller dans les magasins Up ; cours de cuisine au Lafayette Maison). Les clients peuvent personnaliser les objets achetés comme ils le désirent (Build-A-Bear). On fait en sorte que le magasin devienne un lieu de rassemblement et de rencontre. Il s'agit d'organiser des rencontres avec des personnes reconnues dans la discipline (mini-concerts de la Fnac, DJ du Lafayette VO) mais aussi de faire en sorte que le point de vente soit un lieu de rassemblement et de rencontre entre des individus partageant une passion ou un centre d’intérêt (Harley Davidson)
Faire converger les canaux de commercialisation - Générer de la connaissance client ne signifie pas seulement gérer des flux d'information verticaux (e.g. faire remonter de l'information sur les attentes des consommateurs) mais aussi gérer les flux d'information horizontaux (e.g. diffuser cette information au sein des canaux de distribution pour mieux servir le client). Cette approche permet de renforcer l'organisation autour du client dans la mesure où elle permet de servir le client n'importe où et n'importe quand (Bagge, 2007). Pour y parvenir, il convient de passer d'une vision technicienne à une vision client du multi-canal où les assortiments sont pensés de façon cohérente, non pas pour éviter les effets de cannibalisation mais au contraire, pour faciliter la circulation des clients d'un canal à l'autre. Cette approche nécessite aussi de développer une vision et une gestion intégrée des canaux de distribution et par conséquent, de repenser les ratios pour évaluer la performance - tels que le chiffre d'affaires au mètre carré ou le taux de transformation - qui ne sont pas adaptés et entretiennent une concurrence entre les canaux (Bagge, 2007).

\section{Conclusion}

Après avoir mis en évidence les éléments constitutifs des business models dans la distribution, en avoir dressé une première typologie, nous avons exploré les caractéristiques des deux BM identifiés. Notre typologie des business models dans la distribution repose sur des analyses convergentes menées par de nombreux experts. Cela dit, nul doute qu'elle puisse être améliorée dans le futur. Le premier prolongement consisterait à explorer la possibilité d'un ou plusieurs autres BM, comme le propose Noble et al. (2002) en distinguant le BM marché (qui correspond plus ou moins à notre BM-Y), le BM production et le BM vente (qui correspondent plus ou moins à notre BM-X). Au-delà de cette première suggestion, chaque BM pourrait être décomposé en plusieurs combinaisons logiques de RCOA (Ressources, Compétences, Offres, Activités) générant des flux de revenus et les charges associées. Par exemple, dans le domaine de l'équipement du foyer, si l'on considère Darty, le service après-vente constitue une combinaison cohérente de RCOA qui procure à cette enseigne un flux de revenus spécifique, y compris autour de produits qui n'ont pas été vendus dans l'enseigne. Cette approche qui consisterait à décomposer le BM en combinaisons de RCOA permettrait d'enrichir notre compréhension de la diversité des BM, au-delà de la typologie présentée. Pour finir, un examen attentif de l'Enquête Annuelle Entreprise (EAE) menée par l'INSEE permettrait probablement de dégager une typologie quantitative des BM au niveau de secteurs, puis au niveau d'entreprises, une fois les fichiers détails mis à disposition des chercheurs. 


\section{Références}

O. Badot et M. Benoun, Commerce et distribution : prospective et stratégies, Economica, 2005.

C. Barber et B. Tietje, «A distribution services approach for developing effective competitive strategies against "big box” retailers », Journal of Retailing and Consumer Services, No. 11, p. 95-107, 2004.

D. Bagge, « Multi-channel retailing: the route to customer focus », European Retail Digest, No. 53, p.57-70, 2007.

M. Benoun et M.L. Héliès-Hassid, Distribution - Acteurs et stratégies, Economica, Paris, 2003.

B. Berman et J. Evans, Retail management - a strategic approach, Pearson Education International, Upper Saddler River, 2004.

R. Bolton, D. Grewal et M. Levy, « Six strategies for competing through service: an agenda for future research », Journal of Retailing, Vol. 83, No. 1, p. 1-4, 2007.

W. Cascio, « Decency means more than "always low prices”: a comparison of Costco to WalMart's Sam's Club », Academy of Management perspectives, Vol. 20, No. 3, p. 26-37, august 2006.

D. Dion, « A theoretical and empirical study of retail crowding », European Advances in Consumer Research, Vol.4, p. 51-57, 1999.

D. Dion et I. Sueur, La distribution, Dunod, Paris, 2006.

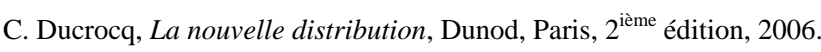

M. Filser, V. des Garets et G. Paché, La distribution : organisation et stratégie, éditions EMS, 2001.

R. Gulati et D. Kletter, « Shrinking core, expanding periphery: the relational architecture of high-performing organizations », California Management Review, Vol. 47, No. 3, p.77104, spring 2005.

P. Hetzel, Planète conso, marketing expérientiel et nouveau univers de consommation, Editions d'Organisation, 2002.

M. Hurt et S. Hurt, « Transfer of managerial practices by French food retailers to operations in Poland », Academy of Management Executive, Vol. 19, No. 2, p. 36-49, 2005.

INSEE, Enquête annuelle d'entreprise, résultats complets pour le commerce, 2004.
H. Isaac et P. Volle, Le commerce électronique, Pearson Education, 2008.

V. Jayaraman et Y. Luo, «Creating competitive advantages through new value creation : a reverse logistics perspective», Academy of Management Perspectives, p. 56-73, may 2007.

S.H. Kim et S.C. Choi, « The role of warehouse club membership fee in retail competition », Journal of Retailing, Vol. 83, No 2, p. 171-181, 2007.

X. Lecocq, B. Demil et V. Warnier, «Le business model au cœur de la croissance de l'entreprise », Expansion Management Review, Vol. 123, p. 96-109, 2006.

R. Lusch, S. Vargo et M. O’Brien, «Competing through service: insights from servicedominant logic », Journal of Retailing, Vol. 83, No. 1, 2007.

P. McGoldrick, Retail marketing, McGraw Hill, Maidenhead, 2003.

B. Moore, « To remain the same, everything has to change », European Retail Digest, No. 50, p.60-62, 2006.

C.H. Noble R.K. Sinha et A. Kumar, «Market orientation and alternative strategic orientations: a longitudinal assessment of performance implications », Journal of Marketing, Vol. 66, October, p. 25-39, 2002.

D. Raff, "Superstores and the evolution of firm capabilities in American bookselling ", Strategic Management Journal, No. 21, p.1043-1059, 2000.

S. Rieunier et P. Volle, Tendances de consommation et stratégies de différenciation des distributeurs, Décisions Marketing, Vol. 27, p. 19-30, 2002.

J. Rowley et L. Haynes, "Customer relationship management: the Matalan way ", The Marketing Review, No. 5, p.175-187, 2005.

R. Sethuraman et A. Parasuraman, "Succeeding in the big middle through technology ", Journal of Retailing, Vol. 81, No. 2, p. 107-111, 2005.

P. Volle, Promotions et choix du point de vente, Dunod, Paris, 1999.

P. Volle, « Produit et information géographique : le géomerchandising », in Le géomarketing, G. Cliquet (ed.), Hermès, Paris, 2002.

G. Voss et K. Seiders, « Exploring the effect of retail sector and firm characteristics on retail price promotion strategy », Journal of Retailing, No. 79, p. 37-52, 2003.

C. Waldman et J.C. Thoenig, De l'entreprise marchande à l'entreprise marquante, Editions d’Organisation, 2005. 